\title{
Research Paper: Behavioral Changes in Combination Therapy of Ethanol and Modafinil on Rats Focal Cerebral Ischemia
}

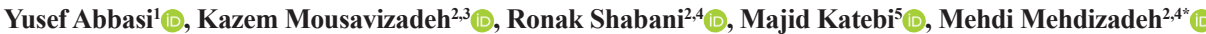

1. Department of Anatomy, Faculty of Medicine, Arak University of Medical Sciences, Arak, Iran.

2. Cellular and Molecular Research Center, Faculty of Medicine, Iran University of Medical Sciences, Tehran, Iran.

3. Department of Molecular Medicine, Faculty of Advanced Technologies in Medicine, Iran University of Medical Sciences, Tehran, Iran

4. Department of Anatomy, Faculty of Medicine, Iran University of Medical Sciences, Tehran, Iran.

5. Department of Anatomy, School of Medicine, Hormozgan University of Medical Sciences, Bandar Abbas, Iran.

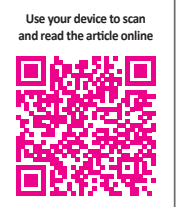

Citation: Abbasi, Y., Mousavizadeh, K., Shabani, R., Katebi, M., \& Mehdizadeh, M. (2020). Behavioral Changes in Combination Therapy of Ethanol and Modafinil on Rats Focal Cerebral Ischemia. Basic and Clinical Neuroscience, 11(3), 269-278. http:// dx.doi.org/10.32598/bcn.11.3.269

http://dx.doi.org/10.32598/ben.11.3.269

Article info:

Received: 15 Sep 2018

First Revision: 05 Oct 2018

Accepted: 16 Feb 2019

Available Online: 01 May 2020

Keywords:

Middle cerebral artery, Ethanol, Neuroprotection, Locomotion, Anxiety

\section{AB S T RA C T}

Introduction: Ethanol is considered as an effective agent in reducing brain stroke injury. In this study, we assessed the effects of modafinil along with ethanol as a combination therapy on behavioral function in Wistar rats.

Methods: The right Middle Cerebral Artery Occlusion (MCAO) was performed and the rats were divided into nine groups ( $\mathrm{n}=8$ per group). The animal groups in this study were as follows: 1. MCAO control group (ischemia without treatment); 2. Vehicle group; 3. Modafinil group that was randomly subdivided into three groups receiving different doses of modafinil $(10,30$, and $100 \mathrm{mg} / \mathrm{kg}$ ) for 7 days before MCAO; 4. Ethanol group receiving $1.5 \mathrm{~g} / \mathrm{kg}$ ethanol at the time of reperfusion; 5. Modafinil + ethanol group that was further subdivided into three groups receiving modafinil at different doses $(10,30$, and $100 \mathrm{mg} / \mathrm{kg}$ ) for 7 days before MCAO and ethanol at the time of reperfusion. The motor behavior was measured using the Garcia test 24, 48, and $72 \mathrm{~h}$ after the ischemia, and the elevated body swing test was performed 48 and 72 $\mathrm{h}$ after the ischemia. The anxiety and locomotor activity were analyzed by open field test 48 and $72 \mathrm{~h}$ post-ischemia.

Results: The results showed that the neurological deficit score, locomotor activity, and unexpected thigmotaxis (anxiety) in the ethanol, modafinil (in a dose-dependent manner), and ethanol+modafinil treatment groups were significantly higher than the MCAO control group.

Conclusion: It seems that the combination therapy of modafinil $(100 \mathrm{mg} / \mathrm{kg})$ and ethanol $(1.5$ $\mathrm{g} / \mathrm{kg}$ ) significantly enhanced neuroprotection via an improvement in locomotor activity and neurological functions.

\section{* Corresponding Author:}




\section{Highlights}

- Combination therapy of modafinil and ethanol enhanced the neuroprotection.

- Modafinil and ethanol increased the anxiety.

- Modafinil and ethanol improved locomotor activity and neurological functions.

\section{Plain Language Summary}

Brain injury following stroke results from the interruption of blood flow to the brain and lack of oxygen in the damaged area. Stroke is the third leading cause of long-term disability and mortality worldwide. People are annually affected by stroke, leading to a broad spectrum of physical and psychological disabilities. There is currently no effective treatment for stroke, and the use of thrombolytic agents is the only available treatment for the management of stroke in patients. Nowadays, research has focused on neuroprotective agents to prevent the worsening of the stroke. Ethanol is readily available, well-tolerated, and easy to use, which its effectiveness has been proven. Light-to-moderate alcohol consumption decreases the mortality and infarct volume following cerebral ischemia. Modafinil has antioxidant properties that its protective effect on epilepsy and Parkinson disease has been confirmed. Unlike ethanol, modafinil improves motor function and boosts locomotor activity leading to its higher neuroprotective effect. The combination of ethanol and modafinil enhanced the synergistic effect on motor function and locomotor activity. The anxiogenic effect was enhanced by the combination of ethanol and modafinil. The infarct size is the main phenomenon often evaluated in experimental studies, but nowadays, the assessment of behavioral functions is being applied to monitor the patients attacked by the stroke. Accordingly, this study aimed at investigating the behavioral changes due to using these compounds as single and combination therapy.

\section{Introduction}

rain injury following stroke results from the interruption of blood flow to the brain and lack of oxygen in the damaged area (Ferdinand \& Roffe, 2016). Tissue Plasminogen Activators (tPA) are currently used to treat acute ischemic stroke. Because of the adverse effects (Nasseri Maleki, Aboutaleb, \& Souri, 2018) and limited therapeutic efficacy, only a few percentages of patients benefit from these types of medications (Ryan, Changya,Tetsuhiro, Xiaokun, Maik, Murali, \& Yuchuan, 2013).

Neuroprotective compounds have been widely developed due to their significant therapeutic effectiveness (Beraki et al., 2013). Ethanol as a neuroprotective agent possesses useful properties, such as availability, being well-tolerated, and easy mode of administration, as well as merely diffusion across the Blood-Brain Barrier (BBB) (Cai, Stevenson, et al., 2016). That light-to-moderate consumption of alcoholic beverages has shown with neuroprotective effects after the ischemia (Cai, Stevenson, et al., 2016; Cai, Thibodeau, et al., 2016; Kochanski et al., 2013; McCarter et al., 2017).
Ethanol has reported with anti-oxidative and anti-inflammatory potentials that are important in reducing ischemic brain damage and improving sensory/motor neurological defects (McCarter et al., 2017). It (1.5 g/ $/ \mathrm{kg}$ ) improved the motor behavior 2 to 28 days after the stroke (Zhang et al. 2012) and reduced the neurological deficits by $48 \%$ at $24 \mathrm{~h}$ post-reperfusion (Ryan et al., 2013). Combination therapy of ethanol $(1.0 \mathrm{~g} / \mathrm{kg})$, hypothermia, and Normobaric hyperoxia can result in a more significant reduction in neurological deficits evaluated $24 \mathrm{~h}$ after $\mathrm{PA}$ administration (Cai, Stevenson, et al., 2016; Cai, Thibodeau, et al., 2016).

Recent studies have demonstrated a surprising correlation between modafinil and neuroprotection (Han, Chen, Liu, \& Zhu, 2018). This compound decreases the fatigue in stroke survivors (Bivard et al., 2017). It was reported that the intraperitoneal (i.p.) administration of modafinil is effective to improve the motor behavior and reduce the ischemic lesion caused by unilateral microinjection of endothelin-1 (ET-1) (Ueki et al., 1993). The infarct size is the primary phenomenon often evaluated in experimental studies, but nowadays, the assessment of behavioral functions are used to monitor the patients with stroke (Bargiotas, Krenz, Monyer, \& Schwaninger, 2012). Numerous behavioral tests have been developed to examine how the stroke induction affects the senso- 
rimotor and cognitive function and the emotional status in animal models (Ingberg, Gudjonsdottir, Theodorsson, Theodorsson, \& Strom, 2015). The current study aimed at assessing the potential neuroprotective and combinatory effects of modafinil and ethanol on behavioral function in the stroke-induced animal models.

\section{Methods}

\subsection{Animal}

In this study, a total of 72 adult male Wistar rats weighing 280-300 g were used. The animals were divided into nine groups ( 8 rats per cage) and housed at room temperature $\left(22-24{ }^{\circ} \mathrm{C}, 45-50 \%\right.$ humidity) and a standard 12 $\mathrm{h}: 12 \mathrm{~h}$ light-dark cycle with free access to food and water.

The rats were used according to the National Institutes of Health Guide for the Care and Use of Laboratory Animals. This research was approved by the Ethics Committee of the Iran University of Medical Sciences (Ethics Code: IR.IUMS.REC 1395.9221313204).

\subsection{Experimental design}

The rats were randomly assigned to nine groups $(n=8)$ as follows:

1. The middle cerebral artery occlusion (MCAO) group (ischemia without treatment); 2. Vehicle group (Veh); 3. Modafinil group, which was divided into three subgroups receiving different doses of modafinil (10, 30, and $100 \mathrm{mg} / \mathrm{kg}$ : M10, M30, and M100, respectively) for 7 days before MCAO; 4. Ethanol group (E) receiving $1.5 \mathrm{~g} / \mathrm{kg}$ ethanol at the time of reperfusion; 5 . Modafinil + ethanol group, which was divided into three subgroups receiving different doses of modafinil for seven days before MCAO and ethanol at the time of reperfusion (10, 30, and $100 \mathrm{mg} / \mathrm{kg}$ : E+M10, E+M30, and E+M100, respectively). The Open-Field Test (OFT) and Elevated Body Swing Test (EBST) were carried out after 48 and $72 \mathrm{~h}$ and the Garcia test was done 24, 48, and $72 \mathrm{~h}$ after the ischemia in all groups of rats.

\subsection{MCAO model}

Rats were anesthetized by the i.p. injection of $10 \%$ chloral hydrate $(400 \mathrm{mg} / \mathrm{kg})$. The right common carotid and the external and internal carotid arteries were exposed through a midline incision in the neck under a surgical microscope (Olympus Szx12). MCAO was performed by the insertion of a silicone coat filament (Doccol Corp., Sharon, MA, USA) via the right external carotid artery until it reached the anterior cerebral artery (Mokudai et al., 2000; Sicard \& Fisher, 2009). The filament was located in the internal carotid artery for $60 \mathrm{~min}$ and then removed. Ethanol $(1.5 \mathrm{~g} / \mathrm{kg})$ was injected immediately after the removal of the obstruction, and the reperfusion was implemented in the $\mathrm{E}$ and $\mathrm{E}+\mathrm{M}$ groups. Modafinil (Dipharma, Milan, Italia) was dissolved in Dimethyl sulfoxide (Bezu, Shanmugasundaram, Lubec, $\&$ Korz, 2016) and then injected for seven days at 8:00 am every day, and the last injection was executed half an hour before the induction of the ischemic model. The body temperature of animals was monitored using a rectal thermometer (Kent Scientific Corporation, Connecticut; USA) and maintained at $37^{\circ} \mathrm{C}$ while a $220 \mathrm{~V}$ lamp was placed next to the animals.

\subsection{Cognitive and behavioral analysis}

\subsubsection{Bederson and Garcia behavioral assessments}

The somatosensory and motor behavior indices were assessed after the induction of ischemia in rats using the Garcia test. Briefly, six items, such as spontaneous activity, symmetry in the movement of the limbs-tail suspension, forepaw extension, climbing the wall of a wire cage, body proprioception, and response to vibrissae touch were measured 24,48 , and $72 \mathrm{~h}$ after ischemia and the total score ranged from 3 to 18 . Higher scores indicated better sensorimotor performance. Items 1-4 (spontaneous activity, the symmetry of movements, the symmetry of the forelimbs, and climbing the wall of wire cage) measured motor performance, and items 5 and 6 (reaction to touch on and response to vibrissae touch) measured sensory function. The day in the horizontal axis and neural defect score in the vertical axis were set to a maximum of 18 .

The Area of Under Curve (AUC) in the form of a trapezoid was calculated every day in different groups (Ghahari, Safari, Joghataei, Mehdizadeh, \& Soleimani, 2014).

For each rat, the AUC is calculated as follows: $\mathrm{AUC}=(\mathrm{NDS} 1+2 \mathrm{NDS} 2+\mathrm{NDS} 3) / 2$

NDS1 $=$ Neural defect score in day 1

NDS2 $=$ Neural defect score in day 2

NDS3 $=$ Neural defect score in day 3 


\subsubsection{EBST test}

To analyze the experimental stroke in animals EBST was conducted using the Borlongan and Sanberg method. The rats were put in a transparent cage and allowed to habituate for $2 \mathrm{~min}$ and attain a neutral position (having all four paws on the floor). Then, the animals were held in the vertical axis, defined as no deviation of more than $10^{\circ}$ to either side. A swing was determined whenever the animal moved its head out of the vertical axis to either the right or left side. Healthy animals approximately swung equally to either side implying no brain dysfunction. Animals with a unilateral cerebral lesion, e.g., ischemic stroke, are expected to present a dominant/biased swing direction (Ingberg et al., 2015).

\subsubsection{OFT}

The OFT is used to measure the locomotion and anxiety for the evaluation of the stress or drug response. Rats were placed in a box ( $80 \mathrm{~cm}^{2}$ chamber, $20 \mathrm{~cm}$ high walls) and the floor was divided into equal squares $(5 \times 5 \mathrm{~cm})$ by $1 \mathrm{~cm}$ wide lines. The animals were positioned somewhere in the box and their behavior was recorded using a video for a specified time. Data were analyzed by the EthoVision XT software using the following parameters: distance moved (distance traveled $(\mathrm{cm})$ ) and the time spent in the peripheral and central zones (Walsh \& Cummins, 1976).

\subsection{Statistical analyses}

Data were analyzed by SPSS software version 22. The One-way Analysis of Variance (ANOVA) was used for comparing different groups followed by a Tukey post-hoc test in the Garcia test, OFT, and EBST. Values are expressed as Mean \pm SEM and $\mathrm{P}<0.05$ was considered statistically significant.

\section{Results}

\subsection{Garcia behavioral assessments}

As described in the somatosensory and motor test (Garcia's index), the AUC was used for the determination of the neurological deficit score in ischemic rats (Figure 1). The neurological deficit score of the E group (15 \pm 5$)$ was significantly higher than the MCAO $(\mathrm{P}<0.001)$ and Veh $(\mathrm{P}=0.034)$ groups. The neurological deficit score of the E+M100 (39.21 \pm 80$)$ group was significantly higher than the E $(15 \pm 5), \mathrm{M} 10(28.33 \pm 2.02)$, and $\mathrm{E}+\mathrm{M} 10$ $(19.5 \pm 1.08)$ groups $(\mathrm{P}<0.0001)$.

\subsection{Elevated Body Swing Test (EBST)}

The EBST and Garcia test were used for the measurement of the neurological deficit (motor function) (Figure $2 \mathrm{~A}$ and B). The proportion of the left-side swings 48 $\mathrm{h}$ after the ischemia (Figure 2A) in the MCAO group $(1 \pm 0)$ did not differ from the Veh group $(1 \pm 0)(\mathrm{P}>0.05)$. However, the proportion of the left-side swings in the $\mathrm{E}+\mathrm{M} 100(0.62 \pm 0.05)$ group was significantly lower than the $\mathrm{E}(0.94 \pm 0.52), \mathrm{M} 10(0.97 \pm 0.05)$, and $\mathrm{E}+\mathrm{M} 10$ $(0.93 \pm 0.5)$ groups $(\mathrm{P}<0.0001)$. The proportion of the left-side swings 72 hours after the ischemia (Figure 2B) was not significant among the groups $(\mathrm{P}>0.05)$.

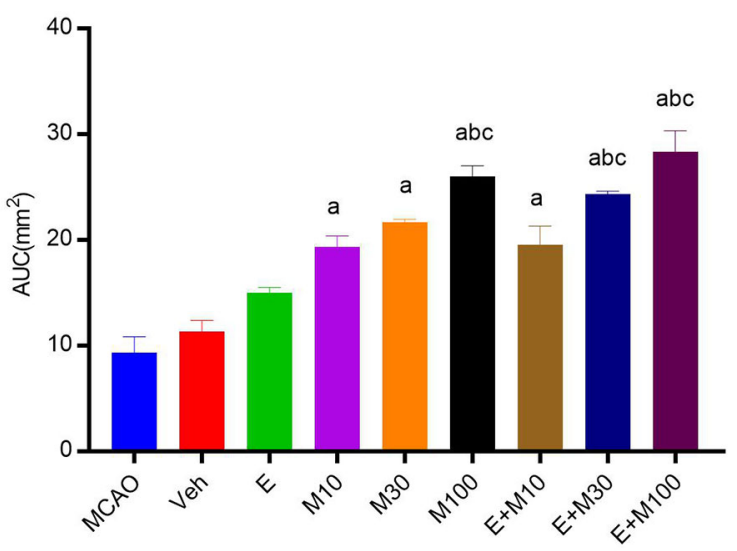

NEUR SCIENCE

Figure 1. Comparison of the neurological deficit scores (AUC) in different groups

(aP<0.05 vs. E); (bP<0.05 vs. E+M10); (cP<0.05 vs. M10);

AUC: Area under curve; Veh: Vehicle; M10: Modafinil (10 mg/kg), M30: Modafinil (30 mg/kg); M100: Modafinil (100 mg/kg); E+M10: Ethanol (1.5g/kg)+modafinil (10mg/kg); E+M30: Ethanol (1.5g/kg)+modafinil (30 mg/kg); E+M100: Ethanol (1.5g/ $\mathrm{kg})+$ modafinil $(100 \mathrm{mg} / \mathrm{kg})$ 
(A)

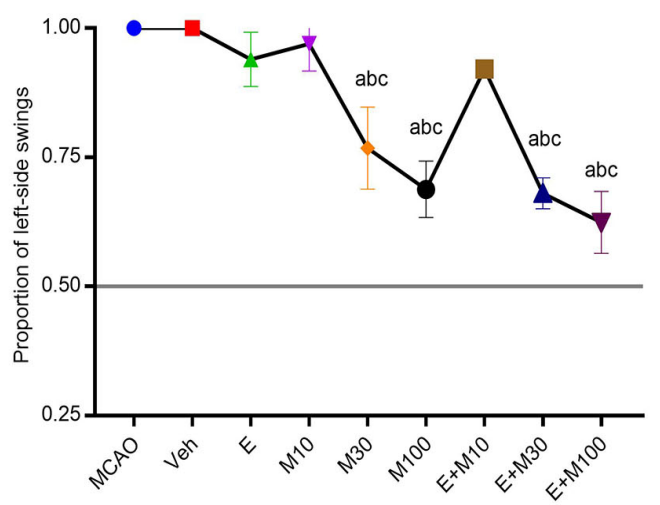

(B)

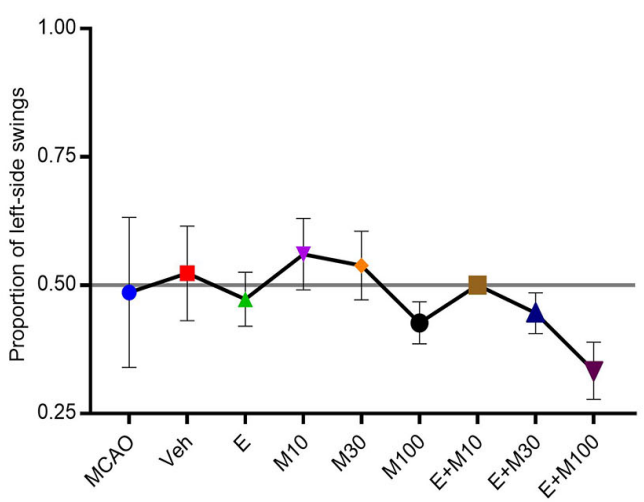

NEUR:SCIENCE

Figure 2. The EBST and Garcia test

A. The Elevated Body Swing Test (EBST) results after 48 and 72 h; B. In different groups;

(aP<0.05 vs. $\mathrm{E}) ;(\mathrm{bP}<0.05$ vs. $\mathrm{E}+\mathrm{M} 10) ;(\mathrm{cP}<0.05$ vs. $\mathrm{M} 10)$; (aP<0.05 vs. $\mathrm{E}) ;(\mathrm{bP}<0.05$ vs. $\mathrm{E}+\mathrm{M} 10)$; $(\mathrm{P}<0.05$ vs. $\mathrm{M} 10)$;

Veh: Vehicle; M10: Modafinil (10 mg/kg); M30: Modafinil (30 mg/kg); M100: Modafinil(100mg/kg); E+M10: Ethanol (1.5 g/ $\mathrm{kg})+$ modafinil (10 mg/kg); E+M30: Ethanol (1.5 g/kg)+modafinil (30 mg/kg); E+M100: Ethanol (1.5 g/kg)+modafinil (100 mg/kg)

\subsection{OFT}

The OFT was used to determine the locomotor activity by the total traveled distance $(\mathrm{cm} / 5 \mathrm{~min})$ assessed in ischemic rats (Figures $3 \mathrm{~A}$ and $4 \mathrm{~A}$ ). The total traveled distance

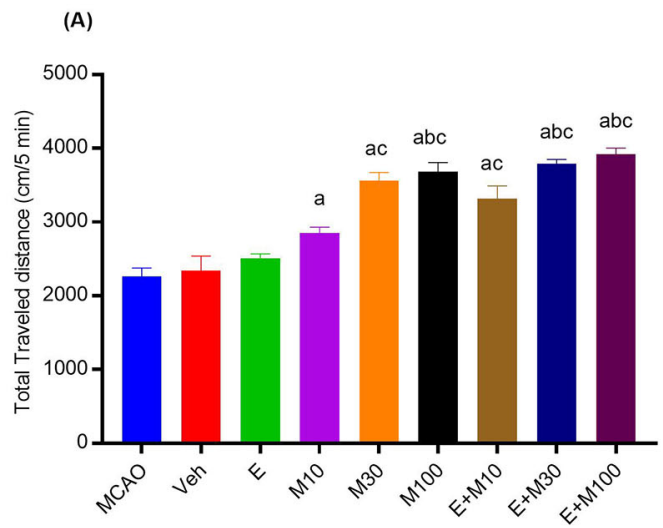

(Figure 3A) by rats in the E group $(2508 \pm 61.15)$ did not significantly differ from the MCAO $(2263 \pm 111.72)$ and Veh (2340 \pm 199.44$)$ groups $48 \mathrm{~h}$ after the ischemia $(\mathrm{P}>0.05)$. However, the total traveled distance in the

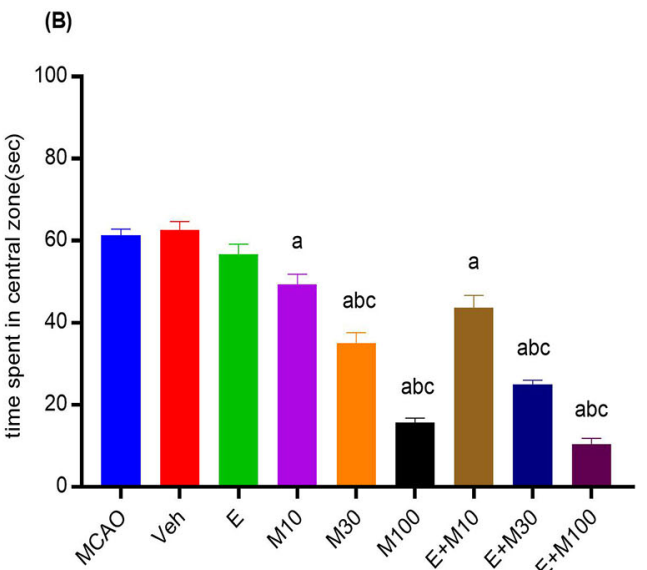

NEUR:SCIENCE

Figure 3. The effects of various doses of modafinil and ethanol $48 \mathrm{~h}$ after ischemia in the open field test in different groups.

A. Total traveled distance (cm/5 min); B. Time spent in central zone (s);

$(\mathrm{aP}<0.05$ vs. $\mathrm{E}) ;(\mathrm{bP}<0.05$ vs. $\mathrm{E}+\mathrm{M} 10) ;(\mathrm{cP}<0.05$ vs. $\mathrm{M} 10)$;

Veh: Vehicle; M10: Modafinil(10 mg/kg); M30: Modafinil(30 mg/kg); M100: Modafinil(100 mg/kg); E+M10: Ethanol(1.5 g/ $\mathrm{kg})+$ modafinil(10 mg/kg); E+M30: Ethanol(1.5 g/kg)+modafinil(30 mg/kg); E+M100: Ethanol(1.5 g/kg)+modafinil $(100$ mg/kg) 
(A)

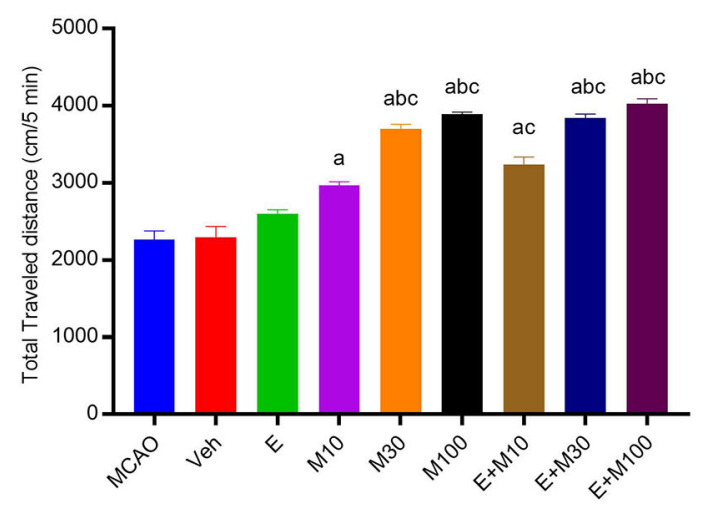

(B)

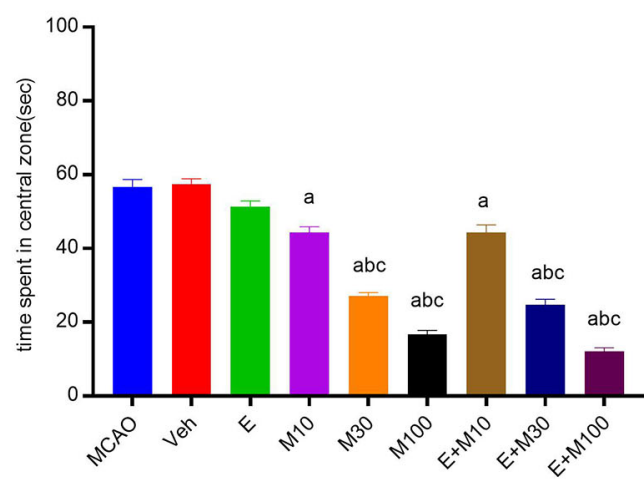

NEUR SCIENCE

Figure 4. The effects of various doses of modafinil and ethanol $72 \mathrm{~h}$ after ischemia in the open field test in different groups

A. Total traveled distance ( $\mathrm{cm} / 5 \mathrm{~min})$; B. Time spent in the central zone (s);

(aP<0.05 vs. E), (bP<0.05 vs. E+M10), (cP<0.05 vs. M10);

Veh: Vehicle; M10: Modafinil(10 mg/kg); M30: Modafinil(30 mg/kg); M100: Modafinil(100mg/kg); E+M10: Ethanol(1.5 g/ kg)+modafinil (10 mg/kg); E+M30: Ethanol(1.5 g/ kg)+modafinil(30 mg/kg); E+M100: Ethanol (1.5 g/kg)+modafinil $(100 \mathrm{mg} / \mathrm{kg})$

E+M100 group ( $3921 \pm 80)$ was significantly higher than the E $(2508 \pm 61.15), \mathrm{M} 10(2850 \pm 81.50)$, and $\mathrm{E}+\mathrm{M} 10$ ( $3317 \pm 171)$ groups $(\mathrm{P}<0.0001)$.

The total traveled distance in the E group $(2603 \pm 48.5)$ was significantly higher than the MCAO $(2266 \pm 111.84$; $\mathrm{P}<0.01)$ and Veh $(2294 \pm 140.76 ; \mathrm{p}<0.01)$ groups $72 \mathrm{~h}$ after the ischemia (Figure 4A). However, the total traveled distance in the E+M100 $(4021 \pm 69)$ group was significantly higher than the E $(2603 \pm 48.5), M 10(2970 \pm 43.92)$, and $\mathrm{E}+\mathrm{M} 10(3236 \pm 100.40)$ groups $(\mathrm{P}<0.0001)$.

The time spent in the central zone was recorded to determine thigmotaxis $48 \mathrm{~h}$ and $72 \mathrm{~h}$ after the ischemia (Figure 3B and 4B). The time spent in the central zone in the E group $(56.66 \pm 2.51)$ was not significantly different from the MCAO $(66.33 \pm 1.52)$ and Veh $(62.66 \pm 2.08)$ groups $48 \mathrm{~h}$ after the ischemia $(\mathrm{P}>0.05$; Figure $3 \mathrm{~B})$. However, the time spent in the central zone in the E+M100 group $(10.33 \pm 1.52)$ was significantly lower than the $\mathrm{E}(56.66 \pm 2.51), \mathrm{M} 10(49.33 \pm 2.51)$, and $\mathrm{E}+\mathrm{M} 10$ (43.66 \pm 3.05$)$ groups $(\mathrm{P}<0.0001)$.

The time spent in the central zone in the E group $(51.33 \pm 1.52)$ was significantly lower than the MCAO $(56.66 \pm 2.08)$ and Veh $(57.33 \pm 1.52)$ groups $(\mathrm{P}<0.05) 72 \mathrm{~h}$ after the ischemia (Figure 4B). However, the time spent in the central zone in the E+M100 ( $12 \pm 1)$ group was significantly lower than the $\mathrm{E}(51.33 \pm 1.52), \mathrm{M} 10(44.33 \pm 1.52)$, and $\mathrm{E}+\mathrm{M} 10$ ( $44.66 \pm 2.08)$ groups $(\mathrm{P}<0.0001)$.

\section{Discussion}

Our results indicated that both single and combination therapy of modafinil (in a dose-dependent manner) and ethanol increased the anxiety but improved neurological function and locomotor activity in ischemia-induced rats. Our previous study showed that both single and combination therapy of modafinil (in a dose-dependent manner) and ethanol decreased apoptosis and increased neuroprotective effect after focal cerebral ischemia (Abbasi, Shabani, Mousavizadeh, Soleimani, \& Mehdizadeh, 2019). Light-to-moderate alcohol consumption decreases the mortality and infarct volume after cerebral ischemia (McCarter et al., 2017).

Cai et al. (2016) and Cai, Thibodeau, et al. (2016) reported that the administration of ethanol as a single $(1 \mathrm{~g} / \mathrm{kg}$ intra-femoral injection) or a combinatory compound with normobaric oxygen and hypothermia reduced the neurological deficits. Wang et al. reported that ethanol at a dose of $1.5 \mathrm{~g} / \mathrm{kg}$ exerted a neuroprotective effect when administrated $4 \mathrm{~h}$ after the onset of ischemia (Zhang et al., 2012), and also improved the neurological function up to $48 \%$ (Ryan et al., 2013) and motor function about 2-28 days after the induction of stroke in rats (Zhang et al., 2012).

McCarter et al. (2017) reported that an 8-week pretreatment with red wine and ethanol $(1.4 \mathrm{~g} / \mathrm{kg} /$ day $)$ through the gavage $24 \mathrm{~h}$ before the induction of cerebral ischemia improved the neurological functionality and enhanced the 
focal cerebral ischemia. In this report, we demonstrated that the single use of ethanol $(1.5 \mathrm{~g} / \mathrm{kg})$ when administrated i.p. reduced the neurological defects; however, this value was much lower when compared with modafinil (in a dose-dependent manner). This finding was consistent with the previous studies; however, it has been shown that a single injection of ethanol does not suffice to improve the neurological function (Figure 1). The neuroprotective effect of modafinil has been studied in animal models of neurodegenerative diseases (Bibani et al., 2012).

Ueki et al. reported that the ischemic striatal injury induced by the microinjections of ET-1 in the rat neostriatum is counteracted by modafinil $(10,30$, and $100 \mathrm{mg} / \mathrm{kg}$; i.p.) in a dose-dependent manner (Ueki et al., 1993). In the present study, the i.p. administration of modafinil (10, 30, and $100 \mathrm{mg} / \mathrm{kg}$ ) improved the neurological function in a dosedependent manner that is in agreement with the previous studies. Notably, the combination therapy of modafinil (10, 30 , and $100 \mathrm{mg} / \mathrm{kg}$; i.p) and ethanol $(1.5 \mathrm{~g} / \mathrm{kg})$ reduced the neurological defect suggesting that the neuroprotective effect of ethanol could be amplified when combined with modafinil. The effect of modafinil is more pronounced in the treatment groups compared with the other groups after MCAO. Modafinil could improve the behavioral deficit and protect the neuron lesion in Parkinson disease models due to its antioxidant activity (Ando et al., 2018).

The current form of EBST was modified by Borlonganand Sanberg as a test for asymmetrical motor behavior in an animal model of ischemic stroke (Borlongan, Cahill, \& Sanberg, 1995). In this report, we observed the complete left side swing after $48 \mathrm{~h}$ in the Veh and MCAO groups. However, other treatment groups, specifically the E+M100 group showed results similar to the normal rats. The left side swing was decreased in all groups and approximately reached the baseline level $(0.5) 72 \mathrm{~h}$ after ischemia. Studies performed on the EBST have reported inconsistent results. In a survey conducted by Ingberg et al., (2015) they showed that after MCAO, rats swung contralateral to the infarct one day after MCAO, but ipsilateral three days after MCAO, when the shift was unexpected.

Another research carried out by Katsumata and colleagues, they reported that after the induction of cerebral ischemia in the right hemisphere of the brain in gerbils while the left-biased swings (contralateral swing) were observed in the day of ischemia-induction followed by ipsilateral side bias on the second day of post-ischemia (Strom, Ingberg, Theodorsson, \& Theodorsson, 2013). Depending on the ischemic region the result of EBST would be different suggesting that the striatal damage results in ipsilateral swing phase but the cortical or com- bined damage would be resulting in contralateral swing phase in animals (Johnston, Dillon-Carter, Freed, \& Borlongan, 2001) Ingberg et al. revealed that there was not a significant difference in groups of animals concerning the proportion of the cortex infarction. According to these studies EBST may not be recommended as a reliable test for the evaluation of the motor asymmetry after MCAO in rats (Ingberg et al., 2015).

Compared with the control mice, treatment with modafinil at the doses of $90-180 \mathrm{mg} / \mathrm{kg}$ could induce hyperlocomotion in mice (Raineri et al., 2011). Edema and inflammation play a significant role in neuronal death and the development of brain lesions after the stroke (Sharma, Westman, \& Nyberg, 1998). Systemic inflammation often leads to a decrease in locomotor activity. Zager et al. reported that modafinil could prevent the increase of Lipopolysaccharide (LPS)-induced inflammation accompanied by the alterations in cell behavior (microglia), the number of infiltration of leukocytes, and rupture of the BBB (Zager et al., 2018).

López-Arnau et al. revealed that mephedrone increased the locomotor activity in rats when combined with ethanol (Lopez-Arnau, Buenrostro-Jauregui, Camarasa, Pubill, \& Escubedo, 2018). In our study, in animals treated with ethanol $(1.5 \mathrm{~g} / \mathrm{kg}$ ), the total traveled distance (locomotion activity at 48 and $72 \mathrm{~h}$ after ischemia) was increased that was lower than the modafinil-treated rats. Modafinil (at the doses of 30 and $100 \mathrm{mg} / \mathrm{kg}$ ) combined with ethanol increased the total traveled distance suggesting that modafinil can promote the locomotion activity. This finding was consistent with the study by Zager et al. and López-Arnau et al., in which they showed that the single use of either ethanol or modafinil along with the combination of these agents could enhance the locomotor activity that may stem from inhibiting GABAergic interneurons that leads to an increase in burst firing of dopamine neurons in the nucleus accumbens (Mitchell et al., 2012).

This effect can also be explained by suppressing the postischemic expression of adhesion molecules and inflammatory mediators, as well as neutrophil infiltration by ethanol and modafinil (McCarter et al., 2017; Zager et al., 2018).

Thigmotaxis is a natural defensive response, in which rats prefer to spend most of their time near the walls and avoid the open center (Lamprea, Cardenas, Setem, \& Morato, 2008). In the OFT, the mice subjected to ischemic stroke tend to spend less time at the center of the arena (Vahid-Ansari, Lagace, \& Albert, 2016). In this report, we demonstrated that the single use of ethanol 
$(1.5 \mathrm{~g} / \mathrm{kg})$ could increase the anxiety; however, it was at a lower level compared with modafinil (in a dose-dependent manner). Ethanol in combination with modafinil also increased anxiety 48 and $72 \mathrm{~h}$ after the ischemia. Modafinil can cause various types of anxiety-altering responses in humans and different animal models. It has been demonstrated that the single doses of modafinil cannot decrease anxiety responses in the rhesus macaque monkey. However, it causes the anxiolytic response in marmoset monkeys (Callithrix jacchus) after a single $(50-225 \mathrm{mg} / \mathrm{kg})$ oral dose of modafinil. Modafinil did not increase panicogenic behaviors in male Swiss albino mice (Mus musculus). These studies indicate the contradictory effect of modafinil on anxiety in different animal models (Johnson \& Hamilton, 2017).

Modafinil administration decreased GABA concentrations and increased glutamate concentrations in the medial preoptic area and the posterior hypothalamus. Administration of GABAA receptor agonists and GABAA receptor antagonists into the hypothalamus of rats decreased and increased anxiety, respectively. Modafinil changed the activity of the frontal cortex and hippocampus that play important roles in anxiety (Randall, Shneerson, Plaha, \& File, 2003). Also, modafinil increased 5-hydroxytryptamine (5-HT) release from the frontal cortex and amygdala (Ferraro, Fuxe, Tanganelli, Tomasini, Rambert, \& Antonelli, 2002) that is associated with an increase in anxiety (Randall et al., 2003).

The anxiolytic and anxiogenic effect of ethanol is almost dose- and species-dependently. Lower doses of ethanol $(1 \mathrm{~g} / \mathrm{kg})$ produced anxiolytic effects, whereas its higher doses $(2.5$ and $3 \mathrm{~g} / \mathrm{kg}$ ) were found to be sedative in adolescent rats (Sakharkar, Tang, Zhang, Chen, Grayson, \& Pandey, 2014). Correspondingly, other studies have shown that ethanol $(1 \% \mathrm{v} / \mathrm{v})$ decreased anxiety in zebrafish (Johnson \& Hamilton, 2017). Besides, ethanol caused withdrawal symptoms, such as anxiety, depression, insomnia, and autonomic hyperarousal (Krystal \& Tabakoff, 2002). The lateral habenula (LHb) is a part of the dorsal posterior thalamus (epithalamus) adjacent to the third ventricle. LHb hyperexcitability is associated with anxiety-like phenotypes. Increased LHb excitability in withdrawal ethanol is due to an increase in glutamatergic transmission and a reduction in M-type potassium channel (Shah et al., 2017). The anxiogenic effect of ethanol $(1.5 \mathrm{~g} / \mathrm{kg})$ was evident in our study 48 and $72 \mathrm{~h}$ after the induction of ischemia, which can be due to ethanol withdrawal leading to increased $\mathrm{LHb}$ excitability.

In conclusion, our findings confirmed that modafinil $(100 \mathrm{mg} / \mathrm{kg})$ in combination with ethanol $(1.5 \mathrm{~g} / \mathrm{kg})$ is beneficial for the recovery of neurologic function and locomotor activity when administered before the induction of stroke. However, these agents have anxiogenic effects at different doses on animal models. Further studies are warranted to illuminate the precise mechanism of modafinil in stroke.

\section{Ethical Considerations}

\section{Compliance with ethical guidelines}

The experiments were conducted according to the $\mathrm{Na}$ tional Institutes of Health Guide for the Care and Use of Laboratory Animals. This research was approved by the Ethics Committee of the Iran University of Medical Sciences (Code: IR.IUMS.REC.1395.9221313204).

\section{Funding}

This study was extracted from the PhD. thesis of Yusef Abbasi in Department of Anatomy, Faculty of Medicine, Iran University of Medical Sciences, Tehran. Also, this study was supported by a Research Grant from the Iran University of Medical Science (Grant No.: 29298).

\section{Authors' contributions}

Resources, supervision, conceptualization: Mehdi mehdizadeh, Yusef Abbasi ; Investigation, Methodology: Yusef Abbasi, Mehdi mehdizadeh, Ronak Shabani, Kazem Mousavizadeh ; Writing-original draft: Yusef Abbasi; Writing-review \& editing: Majid Katebi; Funding acquisition: Research deputy in the medical school of Iran University of Medical Sciences.

\section{Conflict of interest}

The authors declared no conflict of interest.

\section{Acknowledgments}

We thank Sobhan Darou Pharmaceutical Company for providing the pure form of the modafinil.

\section{Reference}

Abbasi, Y., Shabani, R., Mousavizadeh, K., Soleimani, M., \& Mehdizadeh, M. (2019). Neuroprotective effect of ethanol and Modafinil on focal cerebral ischemia in rats. Metabolic Brain Disease, 34(3), 805-19. [DOI:10.1007/s11011-018-0378-0] [PMID]

Ando, R., Choudhury, M. E., Yamanishi, Y., Kyaw, W. T., Kubo, M., \& Kannou, M., et al. (2018). Modafinil alleviates levodopainduced excessive nighttime sleepiness and restores monoamin- 
ergic systems in a nocturnal animal model of Parkinson's disease. Journal of Pharmacological Sciences, 136(4), 266-71. [DOI:10.1016/j. jphs.2018.03.005] [PMID]

Bargiotas, P., Krenz, A., Monyer, H., \& Schwaninger, M. (2012). Functional outcome of pannexin-deficient mice after cerebral ischemia. Channels, 6(6), 453-6. [DOI:10.4161/chan.22315] [PMID] [PMCID]

Beraki, S., Litrus, L., Soriano, L., Monbureau, M., To, L. K., \& Braithwaite, S. P., et al. (2013). A pharmacological screening approach for discovery of neuroprotective compounds in ischemic stroke. PLoS One, 8(7), e69233. [DOI:10.1371/journal.pone.0069233] [PMID] [PMCID]

Bezu, M., Shanmugasundaram, B., Lubec, G., \& Korz, V. (2016) Repeated application of Modafinil and Levodopa reveals a drug-independent precise timing of spatial working memory modulation. Behavioural Brain Research, 312, 9-13. [DOI:10.1016/j. bbr.2016.06.003] [PMID]

Bibani, R. H., Tench, C. R., George, J., Manouchehrinia, A., Palace, J., \& Constantinescu, C. S. (2012). Reduced EDSS progression in multiple sclerosis patients treated with Modafinil for three years or more compared to matched untreated subjects. Multiple Sclerosis and Related Disorders, 1(3), 131-5. [DOI:10.1016/j. msard.2012.03.004] [PMID]

Bivard, A., Lillicrap, T., Krishnamurthy, V., Holliday, E., Attia, J., \& Pagram, H., et al. (2017). MIDAS (Modafinil in debilitating fatigue after stroke): A randomized, double-blind, placebo-controlled, cross-over trial. Stroke, 48(5), 1293-8. [DOI:10.1161/STROKEAA HA.116.016293] [PMID] [PMCID]

Borlongan, C. V., Cahill, D. W., \& Sanberg, P. R. (1995). Locomotor and passive avoidance deficits following occlusion of the middle cerebral artery. Physiology \& Behavior, 58(5), 909-17. [DOI:10.1016/0031-9384(95)00103-P]

Cai, L., Stevenson, J., Peng, C., Xin, R., Rastogi, R., \& Liu, K., et al. (2016). Adjuvant therapies using normobaric oxygen with hypothermia or ethanol for reducing hyperglycolysis in thromboembolic cerebral ischemia. Neuroscience, 318, 45-57. [DOI:10.1016/j. neuroscience.2016.01.010] [PMID]

Cai, L., Thibodeau, A., Peng, C., Ji, X., Rastogi, R., \& Xin, R., et al (2016). Combination therapy of normobaric oxygen with hypothermia or ethanol modulates pyruvate dehydrogenase complex in thromboembolic cerebral ischemia. Journal of Neuroscience Research, 94(8), 749-58. [DOI:10.1002/jnr.23740] [PMID]

Ferdinand, P., \& Roffe, C. (2016). Hypoxia after stroke: A review of experimental and clinical evidence. Experimental \& Translational Stroke Medicine, 8, 9. [DOI:10.1186/s13231-016-0023-0] [PMID] [PMCID]

Ferraro, L., Fuxe, K., Tanganelli, S., Tomasini, M. C., Rambert, F. A., \& Antonelli, T. (2002). Differential enhancement of dialysate serotonin levels in distinct brain regions of the awake rat by Modafinil: Possible relevance for wakefulness and depression. Journal of Neuroscience Research, 68(1), 107-12. [DOI:10.1002/jnr.10196] [PMID]

Ghahari, L., Safari, M., Joghataei, M. T., Mehdizadeh, M., \& Soleimani, M. (2014). Effect of combination therapy using hypothermia and granulocyte colony-stimulating factor in a rat transient middle cerebral artery occlusion model. Iranian Biomedical Journal, 18(4), 239-44. [DOI:10.6091/ibj.13852.2014] [PMID] [PMCID]

Han, J., Chen, D., Liu, D., \& Zhu, Y. (2018). Modafinil attenuates inflammation via inhibiting Akt/NF-kB pathway in apoE-deficient mouse model of atherosclerosis. Inflammopharmacology, 26(2), 385-93. [DOI:10.1007/s10787-017-0387-3] [PMID]
Ingberg, E., Gudjonsdottir, J., Theodorsson, E., Theodorsson, A., \& Ström, J. O. (2015). Elevated body swing test after focal cerebral ischemia in rodents: Methodological considerations. BMC Neuroscience, 16, 50. [DOI:10.1186/s12868-015-0189-8] [PMID] [PMCID]

Johnson, A., \& Hamilton, T. J. (2017). Modafinil decreases anxiety-like behaviour in zebrafish. Peer], 5, e2994. [DOI:10.7717/ peerj.2994] [PMID] [PMCID]

Johnston, R. E., Dillon-Carter, O., Freed, W. J., \& Borlongan, C. V. (2001). Trophic factor secreting kidney cell lines: In vitro characterization and functional effects following transplantation in ischemic rats. Brain Research, 900(2), 268-76. [DOI:10.1016/ S0006-8993(01)02327-7]

Kochanski, R., Peng, C., Higashida, T., Geng, X., Hüttemann, M., \& Guthikonda, M., et al. (2013). Neuroprotection conferred by post-ischemia ethanol therapy in experimental stroke: An inhibitory effect on hyperglycolysis and NADPH oxidase activation. Journal of Neurochemistry, 126(1), 113-21. [DOI:10.1111/ jnc.12169] [PMID]

Krystal, J. H., \& Tabakoff, B. (2002). Ethanol abuse, dependence, and withdrawal: Neurobiology and clinical implications. In K. L. Davis, D. Charney, J. T. Coyle, C. Nemeroff, \& American College of Neuropsychopharmacology (Eds.), Neuropsychopharmacology: The fifth generation of progress (pp. 1423-1444) Philadelphia, PA: Lippincott Williams \& Wilkins. https:// books.google.com/books?id=BKwkonZwZD0C\&dq

Lamprea, M. R., Cardenas, F. P., Setem, J., \& Morato, S. (2008). Thigmotactic responses in an open-field. Brazilian Journal of Medical and Biological Research, 41(2), 135-40. [DOI:10.1590/ S0100-879X2008000200010] [PMID]

López-Arnau, R., Buenrostro-Jáuregui, M., Camarasa, J., Pubill, D., \& Escubedo, E. (2018). Effect of the combination of mephedrone plus ethanol on serotonin and dopamine release in the nucleus accumbens and medial prefrontal cortex of awake rats. Naunyn-Schmiedeberg's Archives of Pharmacology, 391(3), 247-54. [DOI:10.1007/s00210-018-1464-x] [PMID]

McCarter, K. D., Li, C., Jiang, Z., Lu, W., Smith, H. C., \& Xu, G., et al. (2017). Effect of low-dose alcohol consumption on inflammation following transient focal cerebral ischemia in rats. Scientific Reports, 7, 12547. [DOI:10.1038/s41598-017-12720-w] [PMID] [PMCID]

Mitchell, J. M., O'Neil, J. P., Janabi, M., Marks, S. M., Jagust, W. J., \& Fields, H. L. (2012). Alcohol consumption induces endogenous opioid release in the human orbitofrontal cortex and nucleus accumbens. Science Translational Medicine, 4(116), 116ra6. [DOI:10.1126/scitranslmed.3002902] [PMID]

Mokudai, T., Ayoub, I. A., Sakakibara, Y., Lee, E. J., Ogilvy, C. S., \& Maynard, K. I. (2000). Delayed treatment with nicotinamide (vitamin $B(3)$ ) improves neurological outcome and reduces infarct volume after transient focal cerebral ischemia in wistar rats. Stroke, 31(7), 1679-85. [DOI:10.1161/01.STR.31.7.1679] [PMID]

Nasseri Maleki, S., Aboutaleb, N., \& Souri, F. (2018). Berberine confers neuroprotection in coping with focal cerebral ischemia by targeting inflammatory cytokines. Journal of Chemical Neuroanatomy, 87, 54-9. [DOI:10.1016/j.jchemneu.2017.04.008] [PMID] [PMCID]

Raineri, M., Peskin, V., Goitia, B., Taravini, I. R. E., Giorgeri, S., \& Urbano, F. J., et al. (2011). Attenuated methamphetamine induced neurotoxicity by Modafinil administration in mice. Synapse, 65(10), 1087-98. [DOI:10.1002/syn.20943] [PMID] 
Randall, D. C., Shneerson, J. M., Plaha, K. K., \& File, S. E. (2003). Modafinil affects mood, but not cognitive function, in healthy young volunteers. Human Psychopharmacology, 18(3), 163-73. [DOI:10.1002/hup.456] [PMID]

Ryan, K., Changya, P., Tetsuhiro, H., Xiaokun, G., Maik, H., Murali, G., \& Yuchuan, D. (2013). Neuroprotection conferred by post-ischemia ethanol therapy in experimental stroke: An inhibitory effect on hyperglycolysis and NADPH oxidase activation. Journal of Neurochemistry, 126(1), 113-21. [DOI:10.1111/ jnc.12169]

Sakharkar, A. J., Tang, L., Zhang, H., Chen, Y., Grayson, D. R., \& Pandey, S. C. (2014). Effects of acute ethanol exposure on anxiety measures and epigenetic modifiers in the extended amygdala of adolescent rats. The International Journal of Neuropsychopharmacology, 17(12), 2057-67. [DOI:10.1017/S1461145714001047] [PMID] [PMCID]

Shah, A., Zuo, W., Kang, S., Li, J., Fu, R., \& Zhang, H., et al. (2017). The lateral habenula and alcohol: Role of glutamate and M-type potassium channels. Pharmacology, Biochemistry, and Behavior, 162, 94-102. [DOI:10.1016/j.pbb.2017.06.005] [PMID]

Sharma, H. S., Westman, J., \& Nyberg, F. (1998). Pathophysiology of brain edema and cell changes following hyperthermic brain injury. In H. S. Sharma \& J. Westman (Eds.), Brain function in hot environment, progress in brain research (pp. 351-412). Vol. 115. Amsterdam: Elsevier. [DOI:10.1016/S0079-6123(08)62043-9]

Sicard, K. M., \& Fisher, M. (2009). Animal models of focal brain ischemia. Experimental \& Translational Stroke Medicine, 1, 7. [DOI:10.1186/2040-7378-1-7] [PMID] [PMCID]

Strom, J. O., Ingberg, E., Theodorsson, E., \& Theodorsson, A (2013). Effects of high and low $17 \beta$-estradiol doses on focal cerebral ischemia: Negative results. Scientific Reports, 3, 3111. [DOI:10.1038/srep03111] [PMID] [PMCID]

Ueki, A., Rosén, L., Andbjer, B., Agnati, L. F., Hallström, A., \& Goiny, M., et al. (1993). Evidence for a preventive action of the vigilance-promoting drug Modafinil against striatal ischemic injury induced by endothelin-1 in the rat. Experimental Brain Research, 96(1), 89-99. [DOI:10.1007/BF00230442] [PMID]

Vahid-Ansari, F., Lagace, D. C., \& Albert, P. R. (2016). Persistent post-stroke depression in mice following unilateral medial prefrontal cortical stroke. Translational Psychiatry, 6(8), e863. [DOI:10.1038/tp.2016.124] [PMID] [PMCID]

Walsh, R. N., \& Cummins, R. A. (1976). The open-field test: A critical review. Psychological Bulletin, 83(3), 482-504. [DOI:10.1037/00332909.83.3.482] [PMID]

Zager, A., Brandão, W. N., Margatho, R. O., Peron, J. P., Tufik, S., \& Andersen, M. L., et al. (2018). The wake-promoting drug Modafinil prevents motor impairment in sickness behavior induced by LPS in mice: Role for dopaminergic D1 receptor Progress in Neuro-Psychopharmacology \& Biological Psychiatry, 81, 468-76. [DOI:10.1016/j.pnpbp.2017.05.003] [PMID]

Zhang, X., Zhang, X., Wang, C., Li, Y., Dong, L., \& Cui, L., et al. (2012). Neuroprotection of early and short-time applying berberine in the acute phase of cerebral ischemia: Up-regulated pAkt, pGSK and pCREB, down-regulated NF-kB expression, ameliorated BBB permeability. Brain Research, 1459, 61-70 [DOI:10.1016/j.brainres.2012.03.065] [PMID] 